\title{
Las bases políticas de los Acuerdos de Paz, para la integración social y productiva de las comunidades retornadas de la Vertiente Norte de Guatemala
}

\author{
Victor René Ovando Chán \\ victorovando22@yahoo.com \\ Licenciado en Educación Ambiental, Maestro en Educación con Orientación en Medio Ambiente \\ Centro Universitario de Petén, CUDEP \\ Universidad de San Carlos de Guatemala
}

Fecha de recepción: 14/05/2018

Fecha de aceptación: 25/07/2018

\begin{abstract}
Resumen
El exilio, al igual que el retorno, tuvo un efecto traumático en la vida de miles de familias desplazadas. Los Acuerdos del 8 de octubre de 1992, entre el gobierno y las Comisiones Permanentes -CCPP-, sentaron las bases políticas para el retorno, integradas posteriormente al Acuerdo para el reasentamiento de las poblaciones desarraigadas por el enfrentamiento armado, firmado en Oslo, Noruega, el 17 de junio de 1994. Para la reinserción social y productiva de las comunidades de retornados, se establecieron 4 ejes estratégicos: un retorno colectivo y organizado que tiene como base el cooperativismo, el acceso a la tierra como medio de producción, el aprovechamiento sostenible de los recursos naturales respecto de las áreas donde se les ubicó y la participación activa de la mujer en los procesos de toma de decisiones para el desarrollo económico y social de la comunidad. En este sentido, se hizo un análisis documental desde una perspectiva historiográfica, de manera que se puede interpretar el contexto político en el que se llevó a cabo este proceso de retorno y la realidad, que hoy viven estas comunidades en el departamento de Petén.
\end{abstract}

\section{Palabras clave}

Integración social, cooperativismo, acceso a la tierra, desarrollo sostenible, participación de la mujer.

\begin{abstract}
The exile, like the return, had a traumatic effect on the lives of thousands of displaced families. The Agreements of October 8, 1992, between the government and the Permanent Commissions -CCPP-, laid the political foundations for the return, later integrated into the Agreement for the resettlement of the populations uprooted by the armed conflict, signed in Oslo, Norway. June 17, 1994. For the social and productive reinsertion of returnee communities, 4 strategic axes were established: a collective and organized return based on cooperativism, access to land as a means of production, sustainable use of natural resources with respect to the areas where they are located and the active participation of women in the decision-making processes for the economic and social development of the community. In this sense, we intend to make a documentary analysis from a historiographical perspective, so that we can interpret the political context in which this process of return was carried out and the reality that these communities live in the Petén department.
\end{abstract}

\section{Keyword}

Rural integration, cooperativism, access to land, sustainability development, participation of women 


\section{Introducción}

Escribir sobre el pasado, no es algo que logre interesar a los guatemaltecos, la amnesia histórica, parece haber olvidado todo el dolor y la tragedia que ocasionó el conflicto armado durante 36 años. Tal es así, que los Acuerdos de Paz, han sido ignorados por los últimos cinco gobiernos, con la complicidad de una ciudadanía indiferente, que ha callado, reclamar su cumplimiento.

El olvido de todos esos actos de genocidio y crímenes de lesa humanidad, indican la pasividad de un pueblo, que ahora parece estar más interesado en combatir la corrupción, que en retomar los Acuerdos de Paz, para resarcir a todas aquellas víctimas de la guerra, y en este contexto, encontramos a los grupos de retornados, familias que tuvieron que salir al exilio para salvar la vida, ante un clima de terror y miedo que les había robado la paz.

Su retorno, les impuso el estigma de subversivos y/o guerrilleros, marginándolos de los programas de apoyo e investigaciones científicas, que pudieran analizar la realidad social, económica, política, cultural y ambiental de estos grupos y que les ayuden a construir puntos de apoyo para direccionar su propio desarrollo.

Olvidados en la selva, en zonas muchas veces improductivas y con un latente problema agrario de litigios sobre la tenencia y propiedad de la tierra, han luchado solos, para salir adelante en un país excluyente, sin derechos y condiciones dignas, que les permitan enfrentar con éxito los embates de la globalización.

En estas circunstancias poco alentadoras, se han aferrado al cooperativismo, como un modelo real y optimista de desarrollo. A ello, han recurrido para acceder a la tierra, para conservar, proteger y manejar sosteniblemente los recursos naturales, también, para propulsar la participación de la mujer en los procesos de toma de decisiones.

Sin embargo, no podemos negar la debilidad de cada uno de estos elementos dentro de la política de reasentamiento de las poblaciones de retornados. En este sentido, resulta clave analizar el nivel de integración de estos grupos en comunidades rurales, partiendo desde el cooperativismo, como estrategia de organización y participación democrática.

\section{Métodos}

Por la clase de medios utilizados, la investigación es de tipo documental, con un paradigma interpretativo, que pone en práctica una perspectiva historiográfica, cuyo objetivo fue hacer un estudio bibliográfico y crítico acerca de los textos escritos sobre el cooperativismo, contexto agrario, sostenibilidad y equidad de género, entre otros tópicos. Consecuentemente tuvo un alcance metodológico de tipo descriptivo, en el que se buscó explicar las condiciones socioeconómicas de comunidades de retornados en Petén.

En tanto que, el área geográfica donde procede el análisis de este artículo corresponde a las comunidades de la Vertiente Norte, específicamente sobre cuatro asentamientos de retornados: Nueva Guatemala, Tecún Umán en el municipio de San Francisco, La Unión Maya Itzá en Las Cruces, Valle de la Esmeralda en Dolores y la cooperativa Nuevo Amanecer en el municipio de La Libertad.

\section{Resultados}

\subsection{Del retorno colectivo y organizado a la reinserción social}

De acuerdo con Valdez (2014:219) "el acuerdo firmado entre las Comisiones Permanentes de Representantes de los Refugiados en México-CC-PP y el gobierno de Guatemala el 8 de octubre de 1992, incluyó como primer punto un retorno colectivo y organizado". Para ello, se organizaron desde los campamentos delegaciones que visitaban los lugares de interés, fincas que finalmente eran seleccionadas por líderes de las FAR insertados en las CCPP y cuya estrategia militar era conformar frentes guerrilleros, ante lo incierto de la Firma de la Paz, dada la crisis política que el "serranazo" generó en 1993 (AAP, 1999:458,459; Valdez, 2014: 224). 
Por esta razón, los primeros bloques de retornados quedaron ubicados cerca de puntos fronterizos.

Una vez escogido el lugar de retorno, se organizaban en cooperativas, para obtener primeramente personería jurídica y luego acceder al crédito ante el Fondo Nacional para la Paz -FONAPAZ- para la compra de tierra y que a partir de esta se pudiera impulsar el desarrollo de sus comunidades. Por otra parte, según Van de Vaeren, $(2000: 125,128)$ "un retorno en grupos organizados era la única manera de tener fuerza suficiente para promover sus intereses y lograr sus objetivos". Aunque es de reconocer que no era la única estructura organizativa que integraban los grupos de retorno, también existían asociaciones de promotores de salud, educación y mujeres, lo que no era visto con buenos ojos por el gobierno, pues habría de enfrentarse a grupos de campesinos organizados y politizados.

Dentro de este apartado resulta importante mencionar que los nueve bloques de retorno estuvieron integrados por familias de diferentes campamentos establecidos en México, que llegaron a conocerse estando en Guatemala, además per- tenecían a diferentes etnias como se ha apuntado con anterioridad y que por tanto, la barrera idiomática, cosmogonía y cosmovisión cultural, entrarían en pugna. También, conviene mencionar que desde los diferentes campamentos en Chiapas, Campeche y Quintana Roo "había un trabajo político clandestino y desleal a través de los dirigentes locales que formaron las CCPP, quienes de acuerdo a su ideología política o de conquista atraían a las familias a una de las 3 vertientes" (Valdez, 2014).

Actualmente solo cinco de los nueve grupos de retorno, continúan organizados en cooperativa, lo cual es coincidente con lo que ocurre a nivel nacional donde de 2,095 cooperativas inscritas solo el $46 \%$ de las mismas permanecen activas (ver tabla 1), un fenómeno normal, cuando el presupuesto asignado al Instituto Nacional de Cooperativas -INACOP- durante los últimos cuatro años (20152018) no supera los trece millones de quetzales (Instituto Nacional de Cooperativas -INACOP-, 2016, 2017a, 2018, Ministerio de Economía -MINECO-, 2016).

Tabla 1

Cooperativas inscritas por grupos

\begin{tabular}{|c|c|c|c|c|c|c|c|c|c|}
\hline \multicolumn{3}{|c|}{$\begin{array}{c}\text { Cooperativas inscritas a nivel } \\
\text { nacional }\end{array}$} & \multicolumn{3}{|c|}{$\begin{array}{l}\text { Cooperativas inscritas } \\
\text { en la Región VIII-Petén }\end{array}$} & \multicolumn{4}{|c|}{$\begin{array}{l}\text { Cooperativas } \\
\text { Bloques de retorno Vertiente Norte }\end{array}$} \\
\hline Activas & Inactivas & Total & Activas & Inactivas & Total & Activas & Inactivas & Canceladas & Total \\
\hline 963 & 1,132 & 2,095 & 65 & 61 & 126 & 5 & 1 & 0 & 6 \\
\hline
\end{tabular}

Fuente: Elaboración propia con base en los datos de INACOP, 2017a, 2017b; Valdez, 2014:222.

Estas cifras corroboran, la falta de apoyo al sector cooperativo y la invisibilización del valor que tiene este tipo de asociativismo como motor de desarroIlo, lo cual es entendible cuando desde el Estado y la sociedad "se tiene la percepción errónea que el cooperativismo no tiene un impacto en el crecimiento económico" (Unidad Nacional de la Esperanza -UNE-, 2007: 117). En este sentido, habre- mos de rescatar que las "empresas cooperativas hacen contribuciones valiosas para la reducción de la pobreza, la generación de empleos, además de promover la integración social, la democratización y la recuperación del tejido social" (López y Mora, 2012: 20), lo cual, es importante desarrollar en comunidades como estas, a fin de lograr la igualdad, la paz y la justicia social. 
De las cinco cooperativas aún vigentes (ver tabla 2), el total de ellas están dedicadas a la agroindustria, cuatro de ellas integradas en la Federación de Cooperativas Agroindustriales y de Manejo de Recursos Agroforestales y Naturales -FECAIRAN- y la faltante en la Federación de Cooperativas Agrícolas de Guatemala ${ }^{1}$. Actualmente la organización cooperativa es uno de los principales medios que tienen estas comunidades para impulsar su desarrollo socioeconómico. Sin embargo, "los asociados todavía están buscando cómo consolidar la cooperativa como organización participativa. De momento están atrapadas en un círculo vicioso donde existe reticencia en asumir cargos, por escasez de resultados positivos, debido a la falta de transparencia, administración eficiente y asunción de cargos de mayor responsabilidad". (Van der Vaeren, 2000: 300,301), siendo estos, los factores principales de la inactividad y cancelación de muchas cooperativas en el país.

Sin importar todos estos factores que obstaculizan el desarrollo cooperativo en el país, este tipo de organización social, ha permitido la obtención de réditos monetarios, la participación activa de la mujer, además de promover la sustentabilidad en el manejo de los recursos naturales y de "proteger a los pequeños productores contra los impactos del libre mercado (Bonilla, 2014:69). Asimismo, Bonilla, (2014:68), agrega que "el sistema cooperativista en el país, se está consolidando como un modelo para el desarrollo social y productivo".

Tabla 2

Cooperativas de la Vertiente Norte inscritas y activas

\begin{tabular}{|c|c|c|c|c|c|}
\hline \multirow{2}{*}{ Nombre de la cooperativa/bloque } & \multirow{2}{*}{ Actividad principal } & \multicolumn{3}{|c|}{ Asociados } & \multirow{2}{*}{$\begin{array}{c}\text { Federación o } \\
\text { Asociación }\end{array}$} \\
\hline & & M & $F$ & $\mathrm{~T}$ & \\
\hline $\begin{array}{l}\text { Finca Quetzal Cooperativa Agroindustrial } \\
\text { Unión Maya Itzá RL. Las Cruces, Petén } \\
\text { Registro INACOP: } 8171040 \text {. }\end{array}$ & $\begin{array}{l}\text { Producción, distribución } \\
\text { y venta de productos } \\
\text { agrícolas, pecuaria y } \\
\text { forestas. }\end{array}$ & 122 & 9 & 131 & FEDECOAG \\
\hline $\begin{array}{l}\text { Finca Valle de la Esmeralda. Bloque Nueva } \\
\text { Esperanza. Cooperativa Agroindustrial } \\
\text { Nueva Esperanza RL. Dolores, Petén. } \\
\text { Registro INACOP: } 8171041\end{array}$ & $\begin{array}{l}\text { Cultivo de granos básicos, } \\
\text { tienda de consumo y } \\
\text { ganadería }\end{array}$ & 78 & 26 & 104 & FECAIRAN \\
\hline $\begin{array}{l}\text { Finca Huacutz Cooperativa Agroindustrial } \\
\text { Nuevo Amanecer. R.L. La Libertad, Petén. } \\
\text { Registro INACOP: } 8171045\end{array}$ & $\begin{array}{l}\text { Distribución y venta de } \\
\text { maíz, frijol, ajonjolí, maní y } \\
\text { pepitoria. }\end{array}$ & 108 & 20 & 128 & FECAIRAN \\
\hline $\begin{array}{l}\text { San Martin, Cooperativa Agroindustrial } \\
\text { Nueva Guatemala, Tecún Umán. RL. San } \\
\text { Francisco, Petén. INACOP: } 8171043\end{array}$ & $\begin{array}{l}\text { Cultivo de granos básicos, } \\
\text { tienda de consumo y } \\
\text { transporte. }\end{array}$ & 40 & 21 & 61 & FECAIRAN \\
\hline $\begin{array}{l}\text { Laguna Perdida. Cooperativa } \\
\text { Agroindustrial Nuevo Campeche RL. San } \\
\text { Andrés, Petén. Registro INACOP: } 8171050\end{array}$ & $\begin{array}{l}\text { Producción, transporte, } \\
\text { venta de productos agrícolas }\end{array}$ & 16 & 12 & 28 & FECAIRAN \\
\hline Totales & & 364 & 88 & 452 & \\
\hline
\end{tabular}

Fuente: Elaboración propia con base en los datos de Valdez, (2000:222), INACOP, (2017a)

1 La cooperativa Nueva Esperanza R.L., Nuevo Amanecer, Nueva Guatemala-Tecún Umán y Nuevo Campeche, pertenecen a FECAIRAN, en tanto que la cooperativa Unión Maya-Itzá pertenece a FEDECOAG-. 
El cooperativismo, como una respuesta al acuerdo para el reasentamiento de las comunidades desarraigadas por el conflicto armado, promueve el desarrollo rural, "priorizando elevar la producción de estas áreas (Romero, 2010 citado por Bonilla, 2014)². Asimismo tiene un fuerte potencial para enfrentar los asuntos del desarrollo económico y contribuir para alcanzar los Objetivos de Desarrollo Sostenible en su articulación al Plan Nacional de Desarrollo, K'atun Guatemala 2032, para poner fin a la pobreza, luchar contra la desigualdad y la injusticia.

En 2013 las cooperativas en Guatemala generaban más de 700,000 empleos y más de 3,400 millones de dólares en ingresos anuales, lo que representaba el 7.3\% del Producto Interno Bruto del país (Agencia EFE, 2013); en este sentido, el movimiento cooperativo se define como un dinamizador de la economía para la generación de empleo e ingresos, lo cual es sumamente importante en zonas rurales, especialmente donde el conflicto armado produjo una ruptura del tejido social y donde se manifiestan los mayores problemas sociales y de atraso del país, con altos índices de pobreza y vulnerabilidad ambiental, por lo que la integración social de la población ha sido una tarea difícil.

Actualmente el movimiento cooperativista registra 1, 832, 946 asociados ( $11 \%$ de la población nacional), de los cuales 1, 008,120 (55\%) son hombres y 824,826 (45\%) son mujeres, repartidos en 963 cooperativas activas, 13 federaciones y 1 confederación de cooperativas (INACOP, 2017a). Un análisis entre los años 2008, 2010 y 2017, nos refiere que el cooperativismo ha tenido un crecimiento promedio del $26.6 \%$.

En el caso particular del departamento de Petén, "hacia finales de los años setenta, las cooperativas habían conseguido un desarrollo social y económico apreciable, hasta el grado que las cooperativas de los proyectos colonizadores impulsados por la Empresa de Fomento y Desarrollo de Petén -FYDEP-, parecían confirmarse como alternativa

2 Las cooperativas en Guatemala adquieren importancia, especialmente en la seguridad alimentaria, ya que las dedicadas a la agricultura producen el $67 \%$ de los granos básicos del país ( López y Mora, 2012:24) real y viable para los campesinos" (Van der Vaeren, 2000:87). En una segunda etapa entre 1995-2010 el movimiento cooperativo guatemalteco mostró un desempeño que tiende a su consolidación como opción real de desarrollo a nivel nacional, tanto desde la óptica institucional y política, como social y económica (López y Mora, 2012:33).

Cuenta de ello, las cooperativas no solo han generado fuentes de empleo permanentes y temporales, sino que además han aportado al crecimiento económico del país, tal es así, que muchas de estas se han organizado en consorcios ${ }^{3}$ empresariales, incursionando en nuevos sectores y mercados, así lo afirma López y Mora (2012:42), cuando expone que tres de las federaciones y confederaciones, se han convertido en dueñas del $42 \%$ de las acciones del grupo financiero BANRURAL.

El sector cooperativo guarda una correlación entre el porcentaje de estas y el Índice de Desarrollo Humano -IDH-, es menor en departamentos donde existen menos organizaciones cooperativas; asimismo hay una correlación entre menores niveles de pobreza y la presencia cuantitativa de las cooperativas (López y Mora, 2012:45,47).

No obstante el éxito de las cooperativas, según Van der Vaeren (2000:301) depende principalmente de la implementación de un proceso de toma de decisiones más democráticas, la adopción de una estructura organizativa negociada entre los miembros y apropiadas por ellos y no impuesta desde afuera, un manejo eficiente y una administración financiera transparente, la generación de resultados positivos, tangibles percibidos como retribución a lo que se ha invertido; además se deberá "conseguir mayores oportunidades con la creación de esquemas de interoperación y asociativas, como cadenas productivas" (CONFECOOP, 2008:22), para ello habrá de aprovecharse de la Alianza Cooperativa Internacional -ACl-. Asimismo, se deberá aprovechar la oportunidad que otorga el Plan Alianza para la Prosperidad en el que se tiene

3 Ejemplo de ello son: Hortamaya, Inaplasa, Inpasa, en la industria de productos agrícolas; Inforsa, para productos forestales, y Satyc, para servicios de asistencia técnica y capacitación (Reyes, 2015). 
como objetivo fortalecer y ampliar los mecanismos de atención y apoyo a los retornados (Plan Alianza para la Prosperidad -PAPTN-, Secretaría de Planificación y Programación de la Presidencia -SEGEPLAN-, 2017:7).

\subsection{El acceso a la tierra, base para la inserción productiva}

La problemática de la tierra en Guatemala, tiene su génesis en la política agraria de la colonia, que permitió su concentración en pocas manos, mientras la gran mayoría de la población campesina e indígena, carecía de ella (Martínez, 2001). Desde entonces la tierra se ha constituido en el principal motivo de conflictos, de "luchas que han sido las más constantes, dinámicas y beligerantes" (Programa de las Naciones Unidas para el Desarrollo -PNUD-, 2016:179), fue esta, la causa del derrocamiento de Arbenz, tras haber promulgado en 1952 una reforma agraria, que aspiraba a una distribución más justa de la tierra.

El despojo de la tierra, junto a la explotación esclavista y servil del indígena, ha sido la causa de uno de los episodios más fatídicos en la historia de Guatemala; una guerra civil de más de 36 años, gestada por el cúmulo de conflictos históricos de más de cuatro siglos de despotismo, que van desde el repartimiento colonial, hasta la reducción en las fincas cafetaleras y la eminente invasión norteamericana, que terminaría con la posibilidad de elegir al país, su propio desarrollo. Así, la falta de la tierra se convierte en "un punto de focalización del conflicto armado" (Van der Vaeren: 2000:61), un poderoso factor de descontento, para involucrar a la población campesina en la lucha por la tierra.

Este período del conflicto armado (1960-1996) "conocido por la población indígena, como la 'Gran tristeza' [...], causó más de 200 mil muertos, cientos de miles de refugiados y más de un millón de desplazados" (PNUD, 2016:88), sin embargo, el problema de la tierra aún sigue vigente, con un latifundismo que no solo concentra la mayor cantidad de tierras, sino que además acumula las de mayor potencial productivo. En este ambiente de conflictividad agraria, se planifica el retorno de las poblaciones de refugiados en México y, es a partir del Acuerdo del 8 de octubre de 1992 que la CCPP y el gobierno, plantean un retorno, basado en el acceso a la tierra "dentro de un marco de seguridad jurídica que incluye el uso, propiedad y posesión de este recurso" (Acuerdos de Paz...,1998:64).

De esta manera, los nueve bloques de retorno llegan a Petén, entre los años 1995 a 1998, acceden a la tierra y con ello a los litigios propios de la inseguridad jurídica, sobre su tenencia. Un ejemplo, lo constituye la Cooperativa Nueva Guatemala, Tecún Umán, en el municipio de San Francisco, quien actualmente mantiene un conflicto agrario ante la Comisión Presidencial para la Resolución de Conflictos de Tierra (CONTIERRA) y la Secretaría de Asuntos Agrarios de la Presidencia de la República (SAA), por 54 caballerías de tierras que le fueron entregadas y de las cuales, 5 fueron otorgadas a ganaderos y 15 de ellas no aparecen inscritas en el Registro Nacional de la Propiedad, "como efecto de las limitaciones de esta institución, que ha generado tensiones e incerteza, contradicciones e impresiones en la identificación de las fincas, dado que no existe una coordinación entre el Registro de Información Catastral -RICy el Registro General de la Propiedad -RGP-" (PNUD, 2016, 201:193)

Otras cooperativas como Valle de La Esmeralda, Nuevo Campeche y Unión Maya Itzá, se encuentran ubicadas en diferentes zonas de las áreas protegidas del departamento. Lo cual no es menos conflictivo que lo anterior, considerando que más de las tres cuartas partes (79\%) de los suelos de Petén, son de vocación forestal, por lo que la mayoría de estas comunidades fueron asentadas sobre suelos muy pobres para la producción agrícola (Organización de las Naciones Unidas para la Agricultura y la Alimentación -FAO-, citada por Macz y Grümberg, 1999:13). En consecuencia, muchos procesos de retorno fueron un fracaso, ya sea porque se les abandonó en tierras improductivas o se les instaló cerca de áreas protegidas, sin brindarles capacitación técnica o asistencia, generando con ello un creciente problema y una presión adicional sobre 
los recursos naturales" (Ybarra, Obando, Grandia y Schwartz, 2009:26), lo que "complejizó aún más la problemática agraria, condicionando el aprovechamiento de la propiedad" (PNUD, 2016:190)4.

En consecuencia, el nuevo proceso de colonización, organizado desde el refugio en México, vino a agudizar aún más el problema de la tierra en Petén ${ }^{5}$, que desde 1959 con la creación del FYDEP, presentaba grandes crisis, con un "proceso de colonización anárquico, que permitió a los ganaderos adquirir hasta 5 caballerías (originalmente 15 caballerías)"6 (SEGEPLAN, 2013:95; Ybarra et al., 2009: 25), y que en complemento con la Ley de Transformación Agraria (Decreto 1551-62) y la Ley de Adjudicación, Tenencia y Uso de la Tierra de El Petén (Decreto 38-71), planteaba la deforestación como indicador de "mejora" para que esta no fuera expropiada (Macz, Grümberg, 1999:59), lo que generó daños irreversibles a los ecosistemas de la región.

En este contexto, resolver la problemática agraria del país, es fundamental para solucionar la situación de pobreza, pobreza extrema e inequidad de la población que vive en el área rural, por lo que la integración productiva de las poblaciones de retornados, tuvo como eje, el acceso a la tierra, "dado su ubicación eminentemente rural." (Acuerdos de Paz...,1998:65, 123) y el significado que esta tiene para los retornados, para quienes representa no solo un recurso y medio de produc-

$4 \quad$ Esta situación plantea claramente, el incumplimiento de los Acuerdos de Paz, que incluía "estudios sobre la ubicación, régimen legal, extensión linderos y aptitud agrícola de las tierras" (Acuerdos de Paz...,1998:65).

5 Petén ha vivido dos épocas de colonización, la primera, impulsada por la Corona Española, para la reducción de pueblos de indios en el siglo XVII-XIX y la segunda bajo el Estado guatemalteco, impulsado por el FYDEP que inició en 1959 (Schwartz, 2006, citado por SEGEPLAN, 2013: 25)

6 La colonización de Petén ocurrió en el marco de una estrategia de la contrarrevolución, que pretendía redirigir los reclamos de tierra hacia la colonización de tierras bajas (Petén e Ixcán), basado en concepciones coloniales de propiedad de la tierra mediante "mejoras", traducidas en la tala del bosque. Esta es la razón por la cual Petén es el departamento con mayor conflicto agrario del país con 2,401 casos, seguido de Alta Verapaz con 1,079 y Quiché con 871 (PNUD, 2016:118; Ybarra et al. 2009: 55; Macz y Grümberg, 1999:129) ción, sino también el patrimonio familiar principal, condición básica para el re-arraigo (Van der Vaeren, 2000:61).

La ubicación rural de estas poblaciones, pone en perspectiva, el significado de la ruralidad, donde vive la mayoría de la población del país (53.9\%) y del departamento de Petén (68.4\%). Así, "el área rural durante mucho tiempo ha contribuido al crecimiento de la economía nacional, mediante los ingresos y empleos generados en los ámbitos de la agricultura y aprovechamiento de los recursos naturales" (SEGEPLAN, 2009:4; Instituto Nacional de Estadística -INE-, 2013:60). No obstante, el área rural es una de las regiones más abandonadas del país, sin acceso a la tierra y oportunidades de empleo, lo que obliga a la población de estas zonas a migrar como único medio para salir de la pobreza, un problema que afecta a más de las tres cuartas partes de la población petenera (75.1\%) (INE, 2013:60).

De esta manera, el gobierno se plantea apoyar el proceso de reinserción económica y productiva, con programas dirigidos a la población desarraigada como, el Fondo para la Reinserción Laboral y Productiva de la Población Repatriada -FORELAP-, vigente entre 1992 a 1996, y el Programa de asistencia a población desarraigada entre 1998 y 1999 (PNUD, 2016:116). Sin embargo, la vida efímera de estos programas, muy poco pudieron hacer, para establecer un proyecto de desarrollo importante, que permitiera a estas comunidades, superar las condiciones precarias de desnutrición y hambre.

Esto significa, que dichos programas nunca pudieron establecer un modelo de reinserción productiva a través de la tierra, pues "se requería de una política agraria y rural, que pudiera asegurar a los agricultores un precio justo por sus productos, independientemente de la volatilidad del mercado" (Van der Vaeren, 2000:88). Contrariamente, con un modelo de mercados abiertos y una sociedad desigual7, los efectos sobre estas comunidades

$7 \quad$ La concentración de la tierra en Guatemala y su propiedad es el segundo más alto en América Latina, con un índice de Gini de 0.84 (Política Nacional de Desarrollo Rural Integral, 2009:6). En el caso de Petén el índice de Gini en 
fueron negativos, teniendo como resultado la agudización de la pobreza y el detrimento del ambiente (Bonilla y Solís, 2015:69)

La relación entre pobreza y acceso a la tierra, se comprende, cuando el $92 \%$ de los productores agrícolas ocupan el $21.9 \%$ de la fincas censales ${ }^{8}$, en tanto que el $2 \%$ de los productores comerciales ocupan el $65.4 \%$ de la superficie (Asociación de Abogados y Notarios Mayas de Guatemala -ANMAG-, 2017:1; PNUD, 2016:179). Esto significa que son los minifundistas los que producen la mayoría de alimentos que consume el país (Van der Vaeren, 2000:64); en tanto que, "el 43.3\% de la tierra con mayor capacidad productiva, está siendo utilizada para el cultivo de caña de azúcar, café, palma aceitera, hule, pastos para el ganado, lo que repercute fuertemente en la soberanía alimentaria de miles de guatemaltecos ${ }^{10 "}$ (PNUD, 2016:181; Encuesta Nacional Agropecuaria-ENA, 2014).

En consecuencia, la producción alimenticia ha pasado a segundo plano, con "un gobierno cómplice, que coloca el derecho a la propiedad de los grandes terratenientes, por encima del derecho a la alimentación y la vida, lo cual es motivo de grave preocupación" (PNUD, 2016:195)

Petén, no es un caso aislado a lo que acontece en el país, cuando cerca de la mitad (47.7\%) de las familias campesinas, tienen parcelas de menos de 10 manzanas y que dada, la necesidad de descanso

el acceso a la tierra se encuentra entre 0.805 y 0.883 , es decir, muy cerca de 1 (SEGEPLAN, 2013:909)

8 Para el 2003, el 15\% del territorio nacional (1, 642,403 hectáreas) se encontraba sobreutilizada, lo que se traduce en el deterioro del suelo. Contrariamente a ello existe un $37 \%$ de tierra subutilizada, lo que implica que se podría aumentar la producción agrícola, sin causar daños al ambiente (URL, IARNA, 2009:51,52, 61,62)

9 La organización internacional GRAIN citado por el PNUD (2016:195), señala que las fincas pequeñas a nivel mundial suman el $25 \%$ de la tierra agrícola y son estos pequeños productores los que alimentan al mundo.

10 Sayaxché es el municipio de Petén, que más ha experimentado un crecimiento de plantaciones de palma africana, esto ha dado lugar a que comunidades enteras hayan desaparecido, aumentando el problema del campesinado sin tierra, lo cual coincide con la invasión a áreas protegidas (Ybarra et al. 2009:25), que ya han desaparecido en este mismo municipio como la Reserva Biológica San Román. del suelo de manera regular, es difícil que la agricultura en estas parcelas provea la alimentación para la familia. En esta región las microparcelas han aumentado 20\% en un lapso de 10 años (1999-2009). Por otra parte, existe un problema más serio que se ha detectado en este mismo período, el cual indica que el tipo más común de acceso a la tierra por los agricultores en Petén, es el arrendamiento ${ }^{11}$ y que representa un $37 \%$ de la población que los titulares registrados (Ybarra et al., 2009:13,14, 68).

En resumen, a 22 años de los Acuerdos de Paz, las políticas de los gobiernos han ido en detrimento de lo que se ha planteado en estos convenios de Estado, especialmente en lo que se refiere a los Acuerdos de Identidad y Derechos de los Pueblos Indígenas, Reasentamiento de las poblaciones desarraigadas por el enfrentamiento armado y el Acuerdo sobre aspectos socioeconómicos y situación agraria, al señalar "que los temas agrarios y de desarrollo rural, es donde existe principal déficit en el cumplimiento de los Acuerdos de Paz" (SEGEPLAN, 2009:3). Por ejemplo, el compromiso del Estado, de promover la devolución de tierras a poseedores originales y/o buscar soluciones compensatorias, "no hay ningún avance sobre el tema", así lo confirma un informe elaborado por la Misión de Verificación de las Naciones Unidas en Guatemala -MINUGUA- citado por el PNUD (2016:192).

En este sentido, los Acuerdos de Paz han tropezado con el surgimiento del mecanismo de Reforma Agraria Asistida, impulsado por el Banco Mundial (PNUD, 2016:192), En el que las políticas de acceso a la tierra para la población indígena y campesina se han reducido al modelo de mercado de tierra, el que no ha generado los resultados esperados a causa de las imperfecciones del mercado de tierras y otros factores que lo han distorsionado ${ }^{12}$,

11 El arrendamiento en Petén tiene un significado de alquiler o empréstito, cuyo costo varía entre los $0.1,000$ y 1200 quetzales por manzana durante un periodo de 12 meses.

12 El Fondo de Tierra con su modelo de acceso a la tierra vía mercado presenta imperfecciones: a) Falta de claridad en los derechos de propiedad, b) No se reconoce el derecho sobre la tierra de los pueblos indígenas, c) El marco legal que ampara el derecho de la propiedad es inacabado al no existir una legislación agraria sustentativa, d) No existen impuestos sobre la tierra, según categoría productiva, etc. (PNUD, 2016:192) 
reflejado a partir del número de créditos aprobados $^{13}$ para la compra de tierras, la exclusión de las mujeres, la insolvencia técnica y financiera de los proyectos, el desarrollo social y económico de los productores beneficiados, mientras ha aumentado la conflictividad agraria a causa de la deuda. (SEGEPLAN, 2009:8,9)

En Petén, la idea inicial que pretendía la adjudicación de la tierra, mediante la certeza jurídica de la misma, tenía como objetivo: la planificación y uso sostenible de la tierra a largo plazo, el cual fracasó, a partir que los especuladores de tierra aprovecharon las políticas de mejoras, para incrementar el precio de la tierra ${ }^{14}$. Sin embargo, aquellos que han logrado mantener posesión cooperativa de la tierra, han sido más capaces de protegerse de la especulación de tierras ${ }^{15}$ (Ybarra et al., 2009: 27,59).

En conclusión, Guatemala necesita de una reforma del marco jurídico del agro y de un desarrollo institucional en el área rural, que permita un agro más eficiente y más equitativo, que favorezca el acceso a la tierra y la certeza jurídica de la propiedad, que potencialice la eficiencia en el uso de esta (URL, IARNA, 2009:58), para ello se deberá modernizar las modalidades de producción y cultivo, protección del ambiente, seguridad de la propiedad y adecuada utilización de la tierra ${ }^{16}$ (Acuerdos de Paz..., 1998:123, 128).

13 Según el PNUD (2016:193), solo el 1\% de las demandas de tierra han sido satisfechas, por el Fondo de Tierras. De acuerdo con FONTIERRA entre 1998 a 2013 ingresaron 1,740 solicitudes de acceso a la tierra, de las cuales se han adjudicado 265 fincas que corresponde al $15 \%$ de la demanda, beneficiando a 20,187 familias, representado por un monto de Q. 713,107, 618.00

14 El modelo utilizado para la entrega de tierra como propiedad ha generado que la venta de parcelas sea muy fácil y que las tierras destinadas para la producción por pequeños productores, hoy se concentre para ganado o cultivos extensivos (SEGEPLAN, 2013: 96)

15 Las fincas cooperativas a nivel nacional son 6,274 de las cuales el $4 \%$ pertenecen a Petén (228) (SEGEPLAN, 2013:97)

16 Recientemente dentro del problema agrario el Banco Mundial -BM- y el Banco Interamericano de Desarrollo -BID-, promueven la reconceptualización de la actividad agrícola dentro del marco de la nueva ruralidad, promoviendo la diversificación de las formas de sustento del campesino (PNUD, 2016:194)

\subsection{El desarrollo sustentable desde la agricultura familiar en las comunidades de retorno}

En sus orígenes, la postura de los pueblos de Petén ${ }^{17}$, respecto a la tierra, era muy diferente a los pobladores ${ }^{18}$ de otras regiones del país. Tal es así, que estos grupos sociales, tenían una conexión con la naturaleza de integración, orden e interrelación. Así la tierra "no era vista como propiedad privada, sino como un lugar de vida, que contribuía al desarrollo; así que no sentían necesidad de legalizar la propiedad" (Macz, Grümberg, 1999:55, 64). Sin embargo, con el proyecto de colonización, impulsado por el FYDEP en 1959 y la aprobación de la Ley de Transformación Agraria (Decreto 1551-62), generó que las comunidades tradicionales de Petén, se "adaptaran a los valores de la vida de los 'ladinos sureños', pasando por encima de sus costumbres". De hecho, actualmente los "migrantes controlan más tierras que los peteneros nativos" (Ybarra et al., 2009:11).

Lo anterior viene a razón de que los campesinos retornados llegados a Petén, a través de la Vertiente Norte, habían decidido "tumbarlo todo"; no obstante, los compromisos políticos, adquiridos durante el proceso de retorno, impidieron la consumación de esta pretensión; cuya focalización era "cuidar de la selva" y hacer un aprovechamiento racional de los recursos naturales (Van der Vaeren, 2000:27). Asimismo, hay que recordar que muchas de estas comunidades quedaron dentro de áreas protegidas, lo que limitaba el uso de la propiedad, según la categoría de manejo en la que se encontraba adscrita.

En este sentido, observaron su desarrollo, no solo "como la implementación de proyectos productivos y de intercambio económico, sino como [...] el aprovechamiento sostenible de los recursos na-

17 Originalmente los pueblos de Petén eran los Maya Itzá y Mopán, posteriormente se agregaron los Q'eqchíy Ladinos.

18 Para los ladinos sureños la tierra no era un ser con vida y tampoco pensaban que era sagrada, la ven como un medio para conseguir comida para la familia y cuando la pueden legalizar la consideran una propiedad de gran valor (Macz y Grümberg, 1999:65) 
turales que el terreno les ofrecía" (Van der Vaeren, 2000:7). El reto sería, cómo lograr esa interacción ser humano-naturaleza, sin alterar el equilibrio de los ecosistemas naturales, para ello tuvieron que regresar a prácticas ancestrales, como la agroforestería y los huertos mixtos de traspatio.

De esta forma, la agricultura familiar ${ }^{19}$, conocida tradicionalmente como huertos de traspatio, toma un lugar preponderante, para el desarrollo de estas comunidades en el plano de la alimentación y la nutrición, pues además de requerir de espacios muy pequeños, proveía una gran diversidad de productos agrícolas; tal es así que a nivel país produce entre el $27 \%$ y el $67 \%$ del total de la producción de alimentaria" (Díaz, 2017). A este respecto Recalde (2017:93) agrega que en "Guatemala existen más de 1 millón 100 mil pequeños productores en amplios sectores rurales", que a través de prácticas agrícolas amigables con el medio ambiente, promueven la seguridad alimentaria de su comunidad.

Desafortunadamente estas prácticas agroecológicas, no tienen acceso a créditos o asistencia técnica y lo que es peor están expuestos a los efectos del cambio climático y a un modelo de mercados abiertos, que debilita el crecimiento y desarrollo de este tipo de actividades. En consecuencia Recalde (2017:100) propone "unir a los agricultores familiares en cooperativas, para llegar a los mercados, con mayor capacidad de penetración e inserción en cuanto a precios, lo que puede tener un rol importante en el desarrollo económico de Guatemala".

En general, el impulso de "la agricultura familiar, debe ser, sin ninguna duda, el principal programa de desarrollo rural en la lucha contra el hambre y la pobreza. De tal manera que fortaleciendo la agricultura familiar, se pueda contribuir al cumplimiento de los Objetivos de Desarrollo Sostenible ODS"-, ratificados oficialmente por Guatemala en el 2017 (Díaz, 2017:119; Arrecis, 2017, 288).

19 La agricultura familiar, agrupa cerca del $81 \%$ de las explotaciones agrícolas en América Latina y el Caribe; genera el 57 y $77 \%$ del empleo en la región, además de hacer aportes a la seguridad alimentaria y garantizar la sostenibilidad del medio ambiente (Díaz, 2017:103).
En este mismo orden de ideas, es importante resaltar que, según el último informe sobre el cumplimiento de los Objetivos de Desarrollo del Milenio -ODM-20, específicamente en lo referente a garantizar la sostenibilidad del medio ambiente ODM7, Guatemala salió mal calificada en el cumplimiento de las metas planteadas, específicamente en lo que se refiere a: superficie cubierta de bosque, aumento en las emisiones de dióxido de carbono, acceso al agua potable y servicios básicos de saneamiento y mejoramiento de la calidad de vida de las personas que viven en asentamientos precarios urbanos $y$, solo mostró avances significativos en proporción del recurso hídrico utilizado y minimización en la pérdida de la biodiversidad (SEGEPLAN, 2015).

Lo anterior, propone el reto de mejorar los resultados alcanzados en los Objetivos del Milenio -ODM-, al ratificar los ODS y entretejerlo con el Plan Nacional de Desarrollo: K'atún nuestra Guatemala 2032, a partir de la correspondencia que existe entre ambos proyectos $(90 \%$ de coincidencia) (SEGEPLAN, s.f.). Esto trae la esperanza para la aplicación eficiente de la legislación ambiental y el fortalecimiento institucional, para garantizar la seguridad ambiental, mediante la activación de políticas como: la Política Nacional de Cambio Climático (Acuerdo Gubernativo 329-2009), Política Nacional de Desarrollo Rural Integral (Acuerdo Gubernativo 196-2009), Política Agraria (Acuerdo Gubernativo 372-2014) entre otras, además de poder contar con una Ley de Agricultura Familiar, la cual, ya cuenta con dictamen favorable en el Congreso de la República; lo que permitirá encaminar un desarrollo sostenible.

Asimismo, el desarrollo sostenible ya no se debe limitar únicamente a la conservación y protección de los recursos naturales, tal como se pensó, cuando se crearon las áreas protegidas y que limitó a muchas comunidades de retornados a utilizar su propiedad. Hoy estas comunidades bajo un sistema de concesión forestal, han reducido los impactos ambientales en estas zonas, manteniendo la

20 En el 2000 Guatemala se comprometió a alcanzar las 20 metas y 66 indicadores de los ODM (Arrecis, 2017:286). 
capacidad productiva de los ecosistemas y de los bosques (Corzo, 2017; CONAP, 1999).

Sin embargo, no todos los teóricos parecen estar de acuerdo con la idea del desarrollo sostenible, pues lo califican como una propuesta ficticia, señalando que es un concepto sospechoso, al estar al servicio de los intereses de quienes definen el concepto económico y la acumulación de la riqueza. Asimismo, explican que se trata de un término con ambigüedad, además de ser un concepto contradictorio en varios sentidos, pues por una parte, apela a la legitimidad social y política, ya que difícilmente, alguien se opondrá a que el sistema económico funcione de modo tal que no ponga en riesgo el futuro del planeta y por otra, es un concepto que se relaciona con una realidad-el problema ambiental- que resulta urgente e inevitable de atender (Picado, 2010:26, 34)

\subsection{La democratización de las comunidades de retornados a través de la participación de las mujeres exiliadas en el desarrollo económico y social}

Las mujeres fue uno de los sectores más golpeado durante el conflicto armado. Víctimas de brutales torturas y violaciones, fueron objeto de toda clase de vejámenes, que produjeron cambios en su percepción de sí mismas y del mundo. En "algunos casos tuvieron que asumir de manera forzada, un cambio en sus patrones tradicionales de trabajo, haciéndose cargo del trabajo de la tierra, [...] lo que supuso una enorme sobrecarga para las mujeres" (CEH, 1999:77,78).

Su condición de mujer, las puso en un plano de mayor vulnerabilidad, en la que muchas de ellas perdieron hijos, esposos o en el peor de los casos, la vida. La guerra impactó de modo terrible su vida, lo que provocó que muchas salieran al exilio, como una manera de garantizar la sobrevivencia personal y la de su familia.

Las experiencias en el refugio, habían significado para muchas mujeres, nuevas maneras de concebir su rol en la familia a las que tradicionalmente estaban acostumbradas a "cuidar de los hijos, preparar la comida, limpiar la casa" (Pérez y Campillo, s.f.:8; Van der Vaeren, 2000). Incluso fueron ellas, el factor clave para la negociación del retorno y el cumplimiento de los acuerdos de este proceso (Van der Vaeren, 2000:201).

Ahora las mujeres se hallaban organizadas ${ }^{21}$ y demandaban mayor participación social en la toma de decisiones de la comunidad. Por su parte, los hombres consideraban que una vez concluido el retorno, ya no había necesidad de organización y participación de la mujer; sin embargo, las mujeres no estaban dispuestas a regresar a lo de "mujer de casa", por lo que se generó un debate, sobre si debían las mujeres participar en la cooperativa como socios (Van der Vaeren, 2000), la respuesta es que el 19\% (88 de 452 socios ) de las mujeres tuvieron participación dentro de este tipo de organización, aunque en realidad ellas no demandaban tierras, sino que planteaban protegerse ante una eventual separación (divorcio) o viudez.

Actualmente, las mujeres de estas comunidades, están tradicionalmente excluidas de las estructuras de poder y de los procesos de toma de decisiones a nivel comunitario, tal como ocurre en cualquier otra parte del país. El último informe de cumplimiento de los Objetivos de Desarrollo del Milenio -ODM-, específicamente en lo referente a la promoción de la igualdad de género y el empoderamiento de la mujer, indica que a pesar de haberse incrementado su acceso a la educación formal, su incorporación al empleo no agrícola, es limitado22, el analfabetismo alcanza al $48.1 \%$ de las mujeres indígenas y al $18 \%$ de mujeres no indígenas, en tanto que, la participación política de las mujeres ha presentado una evolución positiva (SEGEPLAN,

21 Las mujeres se hallaban organizadas en una estructura llamada "Ixmucané", creada en México por la Coordinadora Mesoamericana para el Desarrollo Integral -COMADEP-, con el propósito de promover la participación activa y reconocida en la toma de decisiones y en la vida comunitaria en general (Van der Vaeren, 200:11)

22 El $45 \%$ de las mujeres no tuvo empleo entre el 2014 y el 2015, con lo que se encuentra por debajo del promedio global en la participación de la mujer en la fuerza laboral (50\% a escala global) (Orozco y Gándara, 2018:6) 
2015); aunque no importante, pues hay que reconocer que la participación política de la mujer, sigue siendo desigual. Petén, por ejemplo, no tiene ninguna funcionaria pública electa popularmente.

A pesar que los Acuerdos de Paz contienen compromisos orientados al empoderamiento de las mujeres, lo que implica el desarrollo de capacidades y habilidades, la generación de ingresos propios y la posibilidad de incidir en los procesos de toma de decisiones y la vida política, en Guatemala las profundas exclusiones y desigualdades han afectado de manera intensa a las mujeres, a quienes no solo se les discrimina, por su condición de mujer, sino también, por ser indígenas y provenir del área rural. (SEGEPLAN, 2010, 2015)

No obstante, el aporte de las mujeres a la economía familiar, es sumamente importante, de hecho, las mujeres producen entre el 60 y el $80 \%$ de los alimentos, además de ser cabeza de hogar del 26\% de las familias ${ }^{23}$ (PNUD, 2016:196; Orozco y Gándara, 2018:6). Su valor es tal, que es la espina dorsal de la cohesión familiar y fundamento de la sociedad. Sin la participación de la mujer, no es posible propender a un desarrollo rural integral, así como al logro de los ODS.

Es esta sociedad de poder patriarcal, propio del pensamiento occidental, la que ha mantenido a las mujeres marginadas al ámbito doméstico, a través de sus relaciones sexuales, emocionales y sentimentales (Herrera, 2012:84); lo que se ve reforzado por políticas públicas que presentan profundos sesgos de género (Pérez y Campillo, s.f.; SEGEPLAN, 2010). En este punto Pérez y Campillo (s.f.) afirma que para superar las desigualdades de género hay que derrumbar falsos postulados que están fuertemente adentrados en la cultura como: el enfoque de género, basado en una concepto cultural, integralidad de las políticas sociales, erradicación del esquema de clases y poder, y planificación de programas de asistencia, para ambos grupos.

23 En América Latina el $25 \%$ de los hogares tienen a una mujer como jefe (Pérez y Campillo, s.f.:7)

\section{Referencias}

AAP (Asociación de Amigos del País). (1999). Historia sinóptica de Guatemala. (Guatemala).

Acuerdos de Paz firmados por el Gobierno de la República de Guatemala y la Unidad Revolucionaria Nacional Guatemalteca (URNG). (1998). (Guatemala). 3ra. Edición. Universidad Rafael Landívar, Instituto de Investigaciones Económicas y Sociales.

Agencia EFE (2013). Más de un siglo de cooperativismo en Guatemala. Agencia de Negocios y noticias. Economía (en línea). Madrid (España).

ANMAG (Asociación de Abogados y Notarios Mayas de Guatemala). (2017). Minifundio y latifundio, un reflejo de la desigualdad en Guatemala. (Guatemala). Boletín Informativo No. 010. NIM-AJPU.

Arrecis, A. (2017). Cambio climático y Objetivos de Desarrollo Sostenible en Guatemala. Revista Análisis de la Realidad Nacional. Edición 21, año 6. (Guatemala). IPSUSAC

Bonilla, G. (2014). Cooperativismo, tierras comunitarias y desarrollo rural. Revista Análisis de la Realidad Nacional. Edición 58, año 3. (Guatemala). IPNUSAC.

Bonilla, G. y O. Solís (2015). "Capitalismo, neoliberalismo y ambiente. Las inconsistencias teóricas de la ciencia económica". Revista Naturaleza, sociedad y ambiente, Volumen 2. Centro Universitario de Sur Oriente, Universidad de San Carlos de Guatemala.

CEH (Comisión para el Esclarecimiento Histórico). (1999). Desplazamiento Forzado en Guatemala Memorias del Silencio (Guatemala). CEH.

CONAP (Consejo Nacional de Áreas Protegidas). (1999). Política nacional y estrategias para el desarrollo del Sistema Guatemalteco de Áreas Protegidas. (Guatemala). CONAP. 
CONFECOOP (Confederación de Cooperativas). (2008). Presencia del cooperativismo en los sectores económicos. (Colombia). Departamento de Análisis de Confecoop.

Corzo, A. (2017). Beneficios socioeconómicos de las concesiones forestales en los grupos comunitarios en Petén. (Guatemala). DIGI/USAC.

Díaz, T. (2017). Agricultura familiar: actor clave del desarrollo rural sostenible. Revista Análisis de la Realidad Nacional. Edición 20, año 6. (Guatemala). IPSUSAC

ENA (Encuesta Nacional Agropecuaria). (2014). República de Guatemala: Encuesta Nacional Agropecuaria 2014. (Guatemala). Instituto Nacional de Estadística.

Herrera, C. (2012). Amor romántico e igualdad de género. Revista Casa de la Mujer, Universidad Nacional de Costa Rica.

INACOP (Instituto Nacional de Cooperativas). (2016). Memoria de Labores 2015. Consejo Directivo de INACOP. (Guatemala).

INACOP (Instituto Nacional de Cooperativas) (2017a). Actualización cuatrimestral del listado de cooperativas, segundo cuatrimestre, Región VIII Petén. (Guatemala). Unidad de Planificación, programación e informática.

INACOP (Instituto Nacional de Cooperativas) (2017b). Registro de Cooperativas. Unidad de Planificación, programación e informática, INACOOP.

INACOP (Instituto Nacional de Cooperativas) (2018). Reportes para la Ley de Acceso a la Información Pública. Sistema de Contabilidad Integrada Gubernamental. Modificaciones internas al presupuesto de ingreso y egresos. (Guatemala).

INE (Instituto Nacional de Estadística) (2013). Mapas de pobreza rural en Guatemala 2011. (Guatemala). INE.
López, B. y A. Mora (2012). Visión panorámica del sector cooperativo en Guatemala: un mecanismo de promoción del desarrollo y lucha contra la pobreza. La Paz (Bolivia). OIT, Oficina de la OIT para los Países Andinos.

Macz, N. y J. Grünberg (1999). Manual de comunidades de Petén. Petén (Guatemala). CARE Guatemala. Cooperación Austriaca para el Desarrollo.

Martínez, S. (2001). La Patria del Criollo. Ensayo de interpretación de la realidad colonial guatemalteca. $2^{a}$. Edición. (México). Fondo de Cultura Económica. Biblioteca presidencial para la paz.

MINECO (Ministerio de Economía) (2016). Acuerdo Gubernativo 263-2016. Presupuesto de Ingresos y egresos 2017. 15 de diciembre 2016. (Guatemala)

Orozco, A. y N. Gándara. (2018). Mujeres tienen poco que celebrar este día. (Guatemala). Prensa Libre

PAPTN y SEGEPLAN (Plan Alianza para la Prosperidad del Triángulo Norte y Secretaría de Planificación y Programación de la Presidencia). (2017). Guatemala, Acciones para la implementación del Plan Alianza para la Prosperidad del Triángulo Norte. (Guatemala). SEGEPLAN.

Pérez, L. y F. Campillo (s.f.). ¿A dónde va la integración de género en el desarrollo rural? Revista Perspectivas Rurales Nueva Época. Universidad Nacional de Costa Rica.

Picado, W. (2016). El desarrollo sustentable como ficción. Una crítica conceptual desde la perspectiva de la historia. Revista Perspectivas: Estudios Sociales y Educación cívica. No.12. Universidad Nacional de Costa Rica.

PNUD (Programa de las Naciones Unidas para el Desarrollo). (2016). Más allá del conflicto, luchas por el bienestar. Informe Nacional de 
Desarrollo Humano 2015/2016. (Guatemala). PNUD.

Recalde, D. (2017). Políticas y estrategias para fortalecer la agricultura familiar guatemalteca. Revista Análisis de la Realidad Nacional. Edición 137, año 7. (Guatemala). IPSUSAC

Reyes, S. (2015, 10 de marzo). Cooperativismo. (Guatemala). Prensa Libre

SEGEPLAN (Secretaría de Planificación y Programación de la Presidencia). (s.f.). Objetivos de Desarrollo Sostenible. (Guatemala). (Trifoliar). SEGEPLAN

SEGEPLAN (Secretaría de Planificación y Programación de la Presidencia) (2009). Política Nacional de Desarrollo Rural Integral. (Guatemala).

SEGEPLAN (Secretaría de Planificación y Programación de la Presidencia). (2010). Igualdad de género y empoderamiento de las mujeres en el marco del cumplimiento de los Objetivos de Desarrollo del Milenio. (Guatemala). SEGEPLAN.

SEGEPLAN (Secretaría de Planificación y Programación de la Presidencia) (2013). Diagnóstico territorial de Petén (Tomo I). Petén 2032, Plan de desarrollo integral de Petén. (Guatemala). SEGEPLAN.

SEGEPLAN (Secretaría de Planificación y Programación de la Presidencia). (2015). Informe fi- nal de cumplimiento de los Objetivos de Desarrollo del Milenio. (Guatemala). SEGEPLAN

UNE (Unidad Nacional de la Esperanza) (2007). Plan de Gobierno; Plan de la Esperanza. Guatemala: UNE.

URL, IARNA (Universidad Rafael Landívar e Instituto de Agricultura, Recursos Naturales y Ambiente)-. (2009). Perfil Ambiental de Guatemala 2008-2009: las señales ambientales críticas y su relación con el desarrollo. (Guatemala). URL/IARNA.

Valdez, M. (2014). Territorio y geopolítica: insurgencia y desmovilización en Petén 1960-2000. Chiapas (México). (Tesis doctoral). Universidad de Ciencias y Artes de Chiapas. Centro de Estudios Superiores de México y Centroamérica.

Van der Vaeren, P. (2000). Perdidos en la selva. Un estudio del proceso de re-arraigo y de desarrollo local de la comunidad Cooperativa Unión Maya Itzá, formada por campesinos guatemaltecos, antiguos refugiados, reasentados en el departamento de Petén. (Guatemala, Amsterdam). Thela Publisher.

Ybarra, M.; O. Obando; L. Grandia y N. Schwartz. (2010). Tierra, migración y vida en Petén, 1999-2009. Municipio de Mixco (Guatemala): Instituto de Estudios Agrarios y Rurales -IDEAR-, Coordinación de ONG y Cooperativas-CONGCOOP 